\title{
Usability of the Certolizumab Pegol Auto-Injection Device in Australian Patients with Chronic Rheumatic Diseases: Results from a Market Research Study
}

Juan Aw'

Hedley Griffiths ${ }^{2}$

Jane Zochling ${ }^{3}$

Alfred Lanzafame ${ }^{4}$

Andrew Jordan ${ }^{5}$

'Peninsula Rheumatology, Langwarrin, VIC, Australia; ${ }^{2}$ Barwon Rheumatology Service, Geelong, VIC, Australia;

${ }^{3}$ Rheumatology Tasmania, Hobart, TAS, Australia; ${ }^{4}$ Immunology, UCB Pharma, Malvern, VIC, Australia; ${ }^{5} \mathrm{BJC}$ Health,

Parramatta, NSW, Australia
Correspondence: Andrew Jordan BJC Health, Level I, |7-2I Hunter St, Parramatta, NSW, 2150, Australia

Tel +6I I 300252698

Fax +6I 0298907655

Email andrew@bjchealth.com.au
Purpose: To determine the usability of the ergonomically designed certolizumab pegol prefilled pen (CZP PFP) in Australian patients with active ankylosing spondylitis, active psoriatic arthritis or moderate-to-severe rheumatoid arthritis.

Patients and Methods: CZP-naive patients were recruited from six clinical centers in Australia between November 2018 and May 2019. Patients and healthcare professionals (HCPs) reviewed training materials and completed pre-injection surveys; patients then selfadministered $\geq 1$ injection with the CZP PFP during each of three visits. Patients and HCPs then completed post-injection surveys. Some survey questions were adapted from the selfinjection assessment questionnaire $\left(\mathrm{SIAQ}_{\mathrm{v} 2.0}\right)$.

Results: Seventy patients participated (65 completed 3 visits); 33 were biologicexperienced. All patients agreed that training materials were informative; $94 \%$ found them easy to understand. Pre-injection, $89 \%$ of patients reported little or no anxiety about having injections; $67 \%$ (79\% in biologic-experienced) were very confident about self-injecting the correct dose with the PFP. Ninety percent of patients were satisfied/very satisfied with their first experience with the CZP PFP; those with pre-injection anxiety reported lower satisfaction ( $43 \%$ vs $79 \%$ "very satisfied"). Confidence and satisfaction increased as visits progressed (for Visit 3 vs Visit 2: 69\% vs 56\% "very convenient"; $75 \%$ vs $67 \%$ "very confident"; $71 \%$ vs $57 \%$ "very satisfied"). All HCPs were confident in their patients' competence to self-inject and thought all patients had overall positive experiences.

Conclusion: Australian patients with chronic rheumatic disease reported high levels of confidence and satisfaction following initial use of the CZP PFP. The availability of devices with patient-centered design innovations may help overcome barriers to self-injection for improved adherence/outcomes.

Keywords: biologic, certolizumab pegol, patient preference, patient satisfaction, selfinjection

\section{Introduction}

Biological disease-modifying anti-rheumatic drugs (bDMARDs), including antitumor necrosis factor (anti-TNFs), are used for the treatment of chronic inflammatory diseases. ${ }^{1}$ Certolizumab pegol (CZP) is the only Fc-free, PEGylated anti-TNF biologic. $^{2,3} \mathrm{CZP}$ is approved in Australia for the treatment of active ankylosing spondylitis (AS), moderate to severe chronic plaque psoriasis, active nonradiographic axial spondyloarthritis (nr-axSpA), active psoriatic arthritis (PsA), and moderate to severe active rheumatoid arthritis (RA). ${ }^{3}$ It is administered 
subcutaneously, and is available for self-injection in a single-use pre-filled syringe or a pre-filled pen (PFP). ${ }^{3}$ For AS, nr-axSpA, RA and PsA, CZP is initiated with a recommended loading dose of $400 \mathrm{mg}$ for adult patients, given as two subcutaneous injections of $200 \mathrm{mg}$ each at Weeks 0,2 and 4, followed by maintenance doses of $200 \mathrm{mg}$ every other week or $400 \mathrm{mg}$ every 4 weeks. $^{3}$

Many patients with chronic rheumatic diseases demonstrate poor medication adherence, limiting the benefits they may derive from treatment. ${ }^{4}$ Self-injection may improve adherence for patients as they can have increased independence and control over the injection process and when the medication is administered, compared with infusion therapy. ${ }^{5,6}$ However, there are barriers to self-injection using a pre-filled syringe, including needle phobia, problems with manual dexterity due to pain and joint swelling in the hands, and a lack of confidence in the self-injection process. ${ }^{5,7}$ User-friendly self-injection devices may overcome some of these challenges, improving treatment outcomes. $^{5}$

The CZP PFP was developed in collaboration with OXO, with input from patients and healthcare providers. ${ }^{6,8}$ It aims to address patients' unmet needs with features such as a button-free delivery system; a wide, non-slip grip to support impaired dexterity; a large viewing window to check medication visually and monitor injection progress; a hidden and retractable needle to reduce the impact of needle phobia; and audible clicks at the start and finish to give patients confidence that a full dose has been administered (Figure S1) ${ }^{1,6,8}$ In a previous comparative usability study, patients with RA ranked the CZP PFP as their preferred device over other anti-TNF PFPs, noting its ease of use and intuitive design. ${ }^{8}$ Results from a patient satisfaction survey conducted in the UK demonstrated that patients had high confidence and satisfaction with self-injection using the CZP PFP. ${ }^{6}$

This market research study aimed to determine the usability of the CZP PFP auto-injection device in Australian patients with active AS, active PsA or moderate to severe RA, including both patients who were biologicnaïve or had previously received a biologic treatment. It explored: patients' perspectives and preferences regarding self-injection; ease of use of the CZP PFP, including, for biologic-experienced patients, comparisons with their previous biologic administration method; the perceived confidence and willingness of patients to use (and continue use of) CZP PFP before and after initial use, including review of the training materials provided with the device; and healthcare professional (HCP) perceptions of patients' confidence levels before and after initial use of the CZP PFP.

\section{Materials and Methods}

\section{Participants}

Eligible patients were at least 18 years of age, had active AS, active PsA or moderate to severe RA diagnosed by a rheumatologist, and were eligible for treatment with CZP on the Australian Pharmaceutical Benefits Scheme, but had not been previously treated with CZP. Patients were excluded if they could not complete the questionnaire on the tablet device, or if they were unable to administer at least one injection by themselves at each study visit. Since this was a market research study, prior approval of the protocol by an ethics committee was not required. The study was conducted in accordance with relevant market research guidelines.

Patients were recruited from six participating clinical centers in Australia. Prospective patients were approached by their rheumatologists and were provided with a Participant Information Sheet and Consent Form. All participants provided written informed consent prior to or at Visit 1.

\section{Study Design}

The study was conducted between November 2018 and May 2019. An overview of the study design is provided in Figure 1. Patients and the HCP (rheumatologists or biologic nurse specialists) responsible for assisting patients completed four sets of short questionnaires throughout their "on-boarding" experience with the CZP PFP, consisting of three visits to clinical centers at Week 0 , Week 2 and Week 4. Loading doses of CZP were administered as two injections at each visit.

At Week 0, patients reviewed the CZP PFP training materials (a video and a step-by-step printed guide). Separate pre-injection surveys were then completed by the patient and the HCP. Following this, the first injection of the CZP PFP was administered, either by the HCP or by the patient themselves. The second injection had to be selfadministered by the patient. After the injections, the patient completed a post-injection survey about their experience using the CZP PFP for the first time, and the HCP completed a survey on their perspective of the participant outcome.

At Week 2, patients self-administered at least one of the two second loading-dose injections with the CZP PFP and 


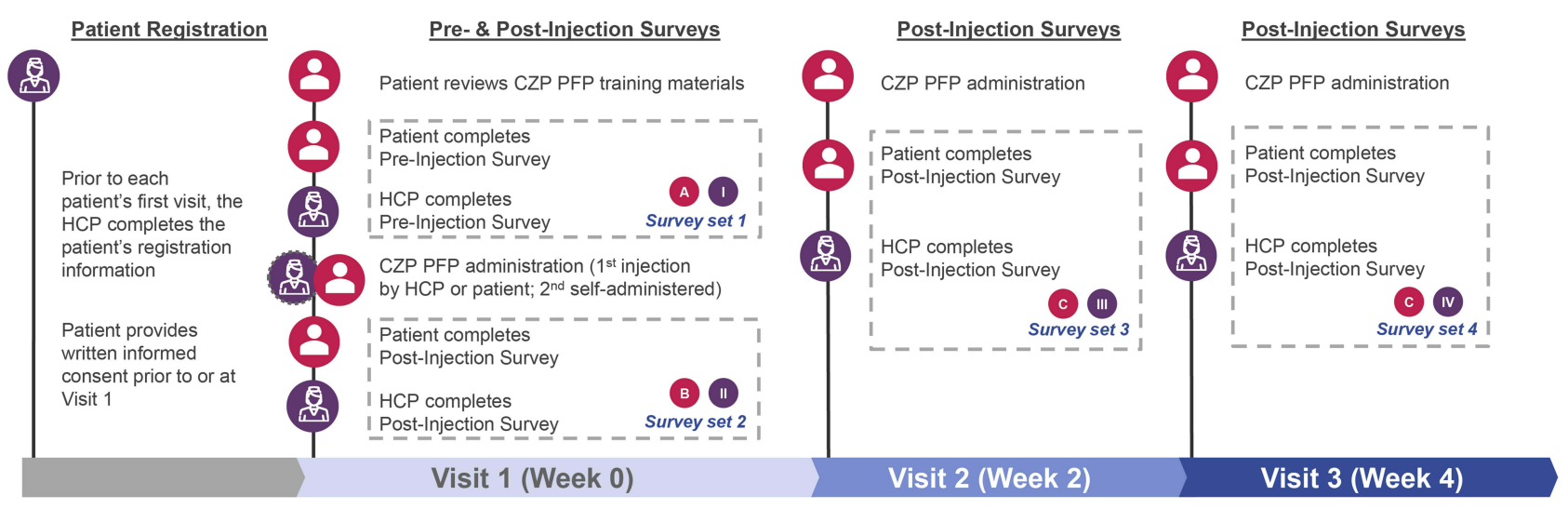

Figure I Overview of study design.

Abbreviations: CZP PFP, certolizumab pegol pre-filled pen; HCP, healthcare professional (doctor or nurse).

completed the second post-injection survey set, which asked about their satisfaction with and convenience of the CZP PFP. HCPs completed a survey about the participants' overall experience using the CZP PFP device and their perception of patients' willingness to continue with self-administration. At Week 4, patients self-administered at least one of the two third loading-dose injections with the CZP PFP, and both the $\mathrm{HCP}$ and the patient completed another set of post-injection surveys similar to that from Week 2 .

\section{Survey Instrument}

Items from the self-injection assessment questionnaire version $2.0\left(\right.$ SIAQ $\left._{\mathrm{v} 2.0}\right)$ were adapted for this study. ${ }^{9}$ To manage the length of the surveys and reduce the burden of participation in research, some domains deemed less relevant to the research objectives were excluded. The pre-injection surveys used in this research included questions on patients' feelings about injections, self-confidence about giving themselves an injection, and feelings about their previous medication (if applicable); post-injection surveys asked about ease of use of the device, satisfaction with self-injection, and willingness to continue to self-inject (Table S1). HCP surveys asked about the nurse or physician's opinion of their patients' confidence and competence to self-inject (Table S2).

\section{Results}

\section{Patient Disposition and Baseline Characteristics}

A total of 70 patients opted to participate in the study, of whom 65 completed all three visits. The mean age of the total opt-in cohort was 53.3 (standard deviation: 15.1) years and the majority were female (71\%; Table 1$)$. About two-thirds were diagnosed with moderate disease $(n=47)$. Almost half of participants were biologicexperienced $(\mathrm{n}=33)$, of whom $42 \%$ were previously treated with adalimumab (Table 1).

\section{Evaluation of Training Materials and Pre-Injection Perceptions}

The majority of patients (94\%) found the training materials quite easy or very easy to understand; all patients agreed that the materials provided all or most of the information they needed (Figure 2). Overall, 77\% of HCPs thought patients had a good understanding of the training materials and $81 \%$ thought patients could confidentially self-inject using the PFP following training. When stratified by prior injection experience, HCPs reported biologic-naïve patients as having lower levels of understanding and confidence (Figure S2).

Patients generally had mild or no anxiety about injections or needles (Figure 3A), regardless of prior biologic experience (Figure S3). Most patients felt confident about self-injection after reviewing the training materials (Figure 3B), though a higher percentage of biologicexperienced patients reported feeling very confident (Figure S3).

\section{Post-Injection Questionnaires}

Two-thirds of patients administered the first injection themselves at the first visit $(\mathrm{n}=46)$, all of whom felt that it was very easy $(n=35,76 \%)$ or quite easy $(n=11$, $24 \%)$ to administer without any help. While most patients $(90 \%)$ reported being satisfied or very satisfied with their first experience using the CZP PFP, those with pre-injection needle anxiety reported lower satisfaction and ease of use scores when compared with patients 
Table I Baseline Demographics

\begin{tabular}{|c|c|}
\hline Characteristics & Opt-in Cohort $(n=70)$ \\
\hline Age, years, mean (SD) & $53.3(15.1)$ \\
\hline \multicolumn{2}{|l|}{ Age, years, n (\%) } \\
\hline 40 and below & $18(26)$ \\
\hline $4 I-50$ & $9(13)$ \\
\hline $5 I-60$ & $19(27)$ \\
\hline $6 I-70$ & $14(20)$ \\
\hline $7 I-80$ & $10(14)$ \\
\hline \multicolumn{2}{|l|}{ Gender, n (\%) } \\
\hline Male & $20(29)$ \\
\hline Female & $50(7 \mathrm{I})$ \\
\hline \multicolumn{2}{|l|}{ Diagnosis, n (\%) } \\
\hline RA & $43(61)$ \\
\hline PsA & $13(19)$ \\
\hline AS & $14(20)$ \\
\hline \multicolumn{2}{|l|}{ Disease severity, $\mathbf{n}(\%)^{\wedge}$} \\
\hline Moderate & $47(67)$ \\
\hline Severe & $23(33)$ \\
\hline $\begin{array}{l}\text { Disease duration, } \\
\text { months, median (range) }\end{array}$ & $63(4-636)$ \\
\hline \multicolumn{2}{|l|}{ Treatment history, n (\%) } \\
\hline Biologic-experienced & $33(47)$ \\
\hline Biologic-naïve & $37(53)$ \\
\hline Previous medication, n (\%) & Of $n=33$ biologic-experienced, \\
\hline Adalimumab & $14(42)$ \\
\hline Etanercept & $5(15)$ \\
\hline Tofacitinib & $3(9)$ \\
\hline Golimumab & $2(6)$ \\
\hline Abatacept & $2(6)$ \\
\hline Secukinumab & $2(6)$ \\
\hline Tocilizumab & $2(6)$ \\
\hline Other ${ }^{\dagger}$ & $3(9)$ \\
\hline
\end{tabular}

Notes: 'Disease severity was defined based on physician diagnosis. ${ }^{\dagger} n=2$ were on "other biologics"; $n=I$ was on a Janus kinase (JAK) inhibitor.

Abbreviations: AS, ankylosing spondylitis; PsA, psoriatic arthritis; RA, rheumatoid arthritis; SD, standard deviation.

who were not anxious about needles (Figure 4A and B). After the first injection, $80 \%$ of patients were comfortable about self-administering their next injection; patients with initial anxiety about needles were more likely to have remaining unease $(35 \%$ versus $3 \%$ in those without needle anxiety; Figure 4C). At Visit 3, $69 \%$ of patients reported finding the CZP PFP "very convenient", while only $27 \%$ of biologic-experienced patients felt that their previous devices were "very convenient" (Figure 4D).
Patients' confidence and satisfaction with self-injection using the CZP PFP increased as visits progressed. A higher proportion of patients reported that the device was "very convenient" at Visit $3(69 \%)$ compared with Visit 2 (56\%; Figure 5). From Visit 2 to Visit 3, more patients also became "very confident to continue selfinjecting" (67\% versus $75 \%)$, "very satisfied with time to inject" (57\% versus $71 \%$ ), and were "very sure the full dose had been given" (73\% versus $83 \%$; Figure 5). By Visit 3, no patients reported finding the CZP PFP "difficult" or "very difficult" to administer; $69 \%$ of patients felt it was "very easy to administer without any help" (63\% at Visit 2; Figure 5).

After observing their patients' first self-administration, $100 \%$ of HCPs agreed that their patients were competent to self-inject; at Week 4, all HCPs were $100 \%$ confident in their patients' ability to continue to self-injections, and thought that all patients had an overall positive experience (Figure S4).

\section{Discussion}

For patients with chronic inflammatory disease who are prescribed subcutaneous anti-TNF therapy, the usability of self-injection devices can have a direct positive impact on medication adherence, which in turn may improve the effectiveness of treatment. ${ }^{4,5-10}$ As non-adherence to medication may be unintentional and driven by difficulties that patients may face when taking their medication, such as dexterity issues or needle phobia, ${ }^{5}$ improvements in medication delivery devices can be associated with higher rates of compliance and persistence. ${ }^{11}$

The CZP PFP was designed with enhanced features to address specific challenges associated with self-injection among patients with impaired dexterity such as RA patients, as well as to address commonly-reported concerns by patients regarding the self-injection process. ${ }^{1,5}$ The aim of this market research was to explore patient and HCP responses to the CZP PFP device through an evaluation of the on-boarding process for CZP selfinjection among a sample of RA, PsA and AS patients in Australia.

Patient training and education have been found to be important factors in improving rates of adherence to selfinjection as they can provide patients with greater confidence and empowerment, and increase levels of independence. ${ }^{5}$ In the UK CZP PFP patient experience study, nurses reported higher levels of confidence in patients' ability to self-inject safely than were reported 


\section{A Ease of understanding}

Step-by-step training guide $(n=70)$

Video guide $(n=70)$

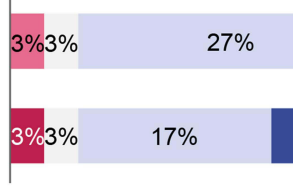

$67 \%$

$\square$ Very difficult to understand $\square$ Quite difficult to understand Neither easy nor difficult to understand $\square$ Quite easy to understand $\square$ Very easy to understand

\section{B Information sufficiency}

Step-by-step training guide $(n=70)$

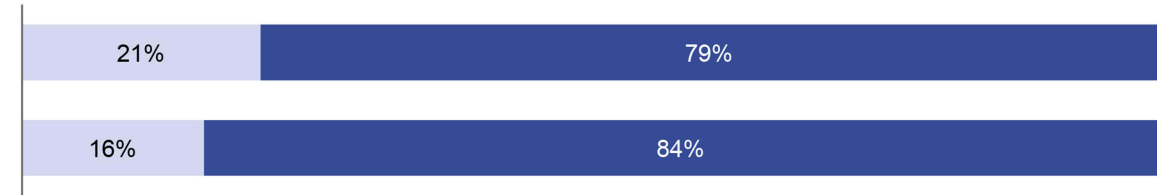

No, NONE of the information I needed was there

SOME of the information I needed was there

no, HARDLY ANY of the information I needed was there

- Yes, ALL the information I needed was here

Yes, MOST of the information I needed was there

Figure 2 Patient evaluations of training materials.

Notes: (A) "Was the information in the step-by-step training guide easy or difficult to understand?"; "Was the information in the training video easy or difficult to understand?" (B) "Was there enough information in the step-by-step training guide to teach you how to self-inject using the [CZP PFP] device?"; "Is there enough information in the training video to teach you how to self-inject using the [CZP PFP] device?"

Abbreviation: CZP PFP, certolizumab pegol pre-filled pen.

\section{A Feelings about injections and needles}

\begin{tabular}{r|r|r|c|c|} 
Feelings about needles $(n=70)$ & $3 \%$ & $6 \%$ & $6 \%$ & $39 \%$ \\
\hline
\end{tabular}

\section{B Confidence about self-injection}

Giving yourself correct dose with self-injecting pre-filled pen $(n=70)$

Giving yourself an injection in a clean and sterile way $(n=70)$

Giving yourself an injection safely $(n=70)$

Giving yourself an injection the right way $(n=70)$

Starting treatment requiring self-injection of medication with pre-filled pen $(n=70)$

\section{- Very hesitant}

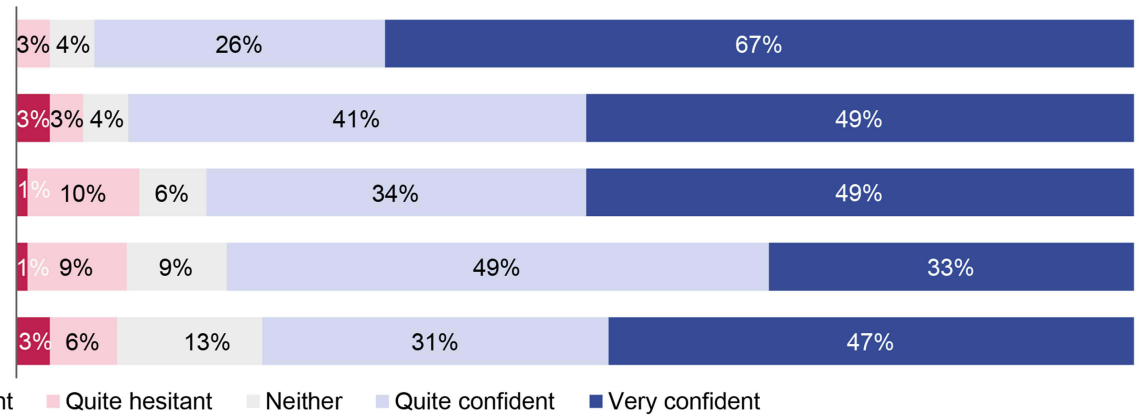

Figure 3 Patients' pre-injection perceptions.

Notes: (A) "In general, how do you feel about needles?"; “In general, how do you feel about having an injection?"; “And how do you feel about giving yourself an injection?" (B) "How confident do you feel about giving yourself the correct dose with a self-injecting pre-filled pen?"; "How confident do you feel about giving yourself an injection in a clean and sterile way?"; "How confident do you feel about giving yourself an injection safely?"; "How confident do you feel about giving yourself an injection in the right way?"; how confident do you feel about starting a treatment for your rheumatoid arthritis/psoriatic arthritis/ankylosing spondylitis that requires you to inject medication with a self-injecting pre-filled pen?.

by the patients themselves. ${ }^{6}$ A similar trend was observed in this sample of Australian patients; after the first visit, $100 \%$ of HCPs were confident about their patients' competence, whereas $20 \%$ of patients had remaining unease regarding further self-injections, particularly in those with pre-injection needle anxiety. As noted by Bailey et al, ${ }^{6}$ this could indicate a gap between the patients' confidence in their abilities and their actual competence to correctly selfinject. This further highlights the importance of adequate training, communication and support at treatment initiation 

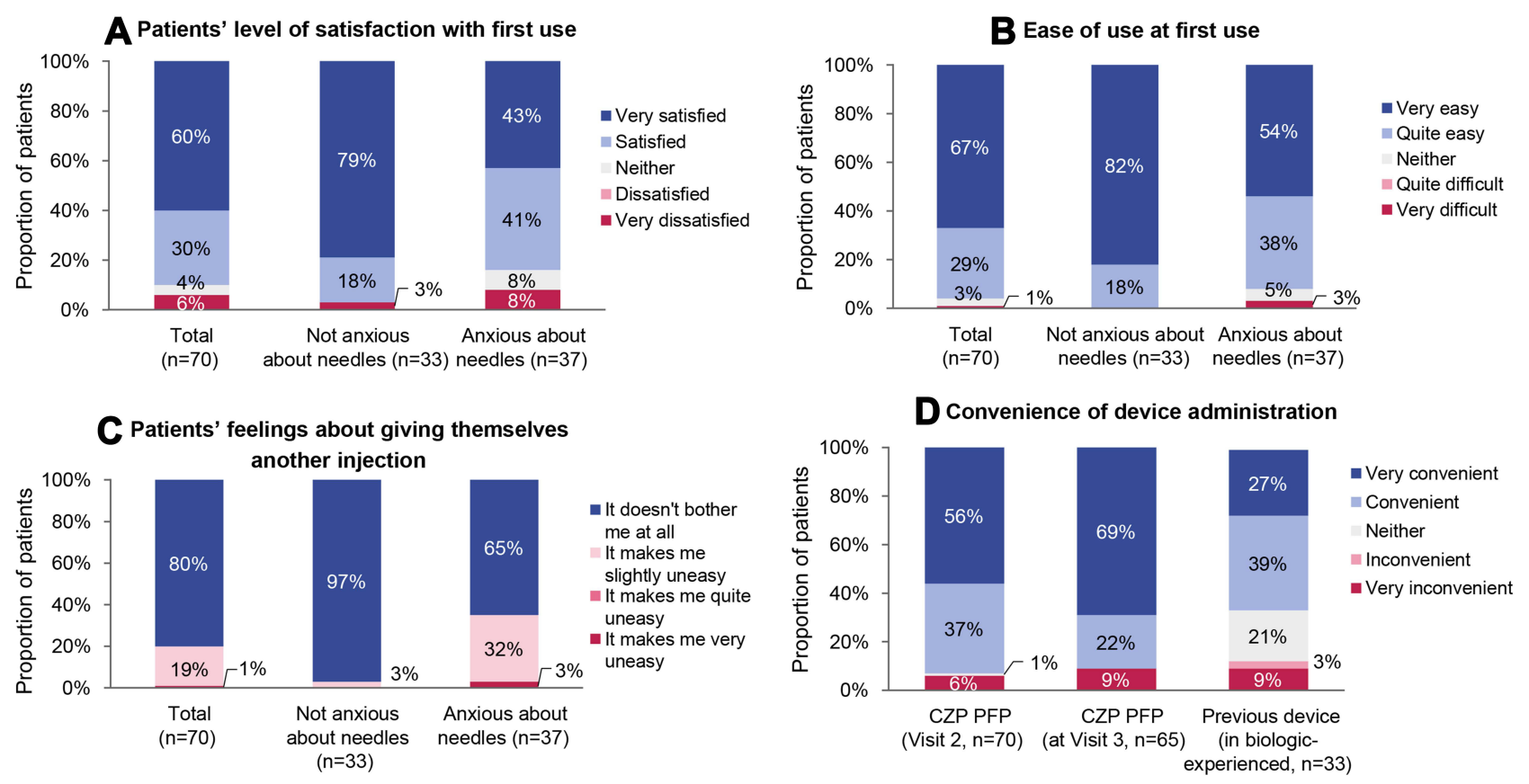

Figure 4 Patients' post-injection perceptions.

Notes: (A) "Overall how satisfied or dissatisfied are you with your first experience using the [CZP PFP] device?" (B) "How difficult or easy was it to use the [CZP PFP] device?" (C) "After giving yourself an injection using the [CZP PFP] device, how do you feel about giving yourself another injection with this self-injecting pre-filled pen?" (D) "Overall how convenient or inconvenient did you find the [CZP PFP] device?"; "How convenient or inconvenient was the way you administered your previous medication?". Abbreviation: CZP PFP, certolizumab pegol pre-filled pen.

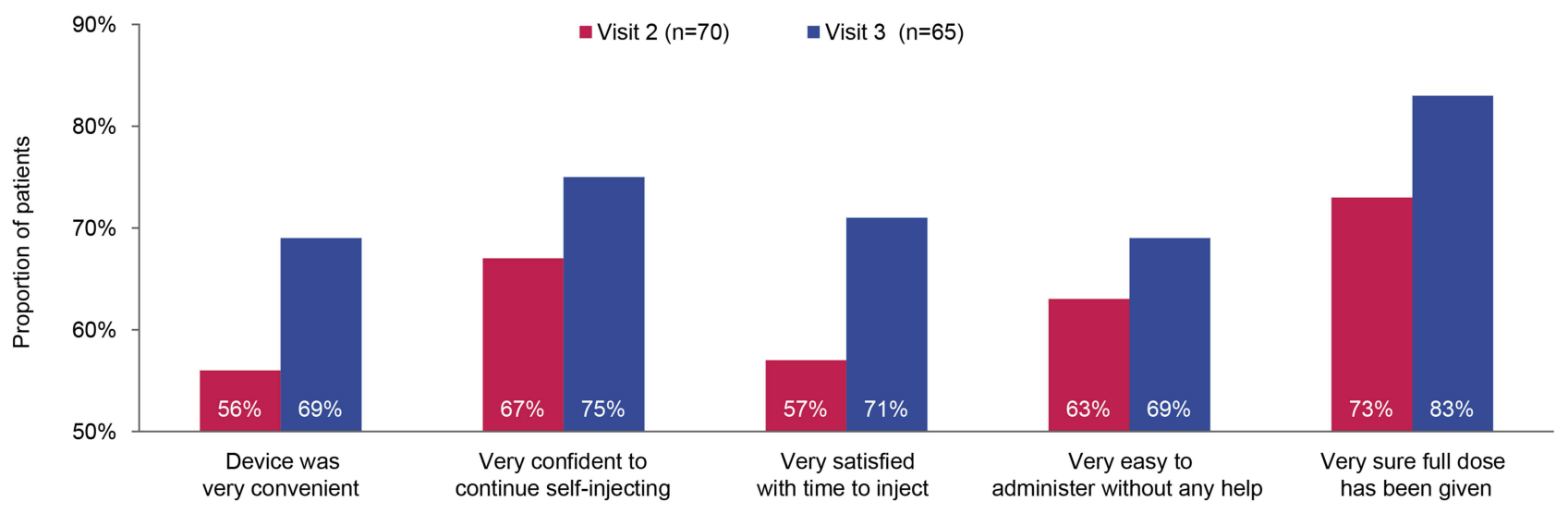

Figure 5 Patient confidence and satisfaction with self-injection at Visits 2 and 3.

Notes: "Overall how convenient or inconvenient did you find the [CZP PFP] device?"; "How confident do you feel about continuing to give yourself the injections at home with the [CZP PFP] device?"; how satisfied are you with the time it takes to inject the medication?; "How difficult or easy was it to administer the injection without any help?"; "How sure are you that you have given yourself the full dose of the injection?"

Abbreviation: CZP PFP, certolizumab pegol pre-filled pen.

in order to reassure patients and manage any injectionrelated anxiety or uncertainty. ${ }^{6,10}$

Needle fear may cause anxiety about self-injections, reducing confidence and negatively impacting the selfinjection process. ${ }^{10}$ The CZP PFP features a hidden retractable needle which remains non-visible to patients during the injection process, aiming to reduce fear related to needle phobia. ${ }^{6}$ However, as demonstrated in the current study, pre-existing fear of needles could still lead to lower satisfaction with self-injection, and higher perception of difficulty at first use of the self-injection device. It is thus particularly important for patients with pre-injection needle anxiety to receive adequate support which acknowledges and manages their emotional response during the injection process. ${ }^{10}$ Comprehensive and easy-to-understand training materials, as well as an intuitive, user-friendly device, can help to overcome this by increasing confidence prior to, during, and after the first self-injection. ${ }^{6,8}$ 
The results from this market research survey builds on previous patient experience research on the device. ${ }^{6,8}$ In a similar market research survey conducted in a UK population of patients with RA, PsA or axSpA, high confidence and satisfaction scores were reported when using the CZP PFP. ${ }^{6}$ This study supports those findings and provides evidence that high levels of patient satisfaction with CZP PFP may also be found among Australian RA, PsA and AS patients.

In a previous comparative usability study, patients with RA ranked the CZP PFP as their preferred device over adalimumab, etanercept, and golimumab PFPs. ${ }^{1,8}$ In these patients who may have impaired dexterity or poor hand strength, features such as an ergonomic handle and buttonfree activation were rated as highly important as it enabled them to hold the device confidently and with a good grip. ${ }^{1,8}$ Likewise, in the current survey, a higher proportion of patients rated the CZP PFP as "very convenient" compared with the administration of their previous medications.

Patients' confidence and satisfaction with self-injection generally improved between Visits 2 and 3, indicating that, with increasing experience, patients felt more comfortable using the CZP PFP. Patient experience studies have reported on the importance of setting up "routines", where the association of self-injection with familiar repeated "rituals" helps to mitigate patients' fear and anxiety by giving patients confidence and control over the administration process. ${ }^{10}$

Although generally high satisfaction scores were reported by the end of the study, it should be noted that a small remaining proportion of patients were not completely satisfied with the CZP PFP. Studies suggest that multiple factors can affect patient satisfaction; ${ }^{5}$ patients often have personal preferences in the treatment of their own disease, and empowering patients to play a more active role in their disease management can improve treatment experience and adherence. ${ }^{5,10}$ To address these differences in patient preferences, a portfolio of self-injection solutions will be needed: for example, some patients may prefer the control provided by the use of a pre-filled syringe, whereas others may prefer some of the functions associated with the PFP. The availability of a range of devices is thus essential in providing choice, and allows patients and physicians to discuss and adapt treatment administration options according to individual needs. ${ }^{5}$

\section{Limitations}

Due to the lack of a comparator in the study, we are unable to comment on direct comparisons of CZP PFP with other selfinjection devices. The SIAQ was amended for this survey, and some domains such as "injection site reactions" or questions within domains were not included in the version used in this study. The exclusion of these elements meant that SIAQ domain scores, which provide a validated evaluation of patients' self-injection experience, ${ }^{9}$ could not be calculated.

Aside from biologics, prior experience with other injected medications may also have impacted patients' expectations of and satisfaction with the CZP PFP; however, a detailed history of non-biologic medications was outside the scope of this market research study. Other factors that were not assessed, such as differences in clinical efficacy or symptom improvement experienced during visits after the first injection (Visits 2 and 3), may also impact overall satisfaction with therapy. ${ }^{10}$

It was a requirement that a $\mathrm{HCP}$ be present during each visit to ensure completion of patient obligations for the market research. It is unclear whether the physical presence of HCPs during self-injection had any impact on patients' perception of and confidence with the CZP PFP. In previous studies, patients have reported that, regardless of the usefulness of the provided training materials, they found the presence of a nurse during their first injection reassuring. ${ }^{6}$

Finally, the study recruited only 70 patients, with 65 patients remaining at Visit 3, and may not be representative of the entire population of Australian CZP PFP patients. In particular, most of the patients opting into the study had mild or no injection or needle anxiety; patients who were most afraid of self-injection may have opted out of the study, reducing the generalizability of these results to the wider patient population.

\section{Conclusions}

Australian patients with AS, PsA and RA generally reported high levels of confidence and satisfaction following their initial use of the CZP PFP. With the provision of holistic training and support, particularly among patients reporting some level of pre-initiation needle anxiety, the availability of a range of devices with patient-centered design innovations could help to overcome barriers to selfinjection, increasing adherence and improving outcomes among patients with chronic rheumatic disease.

\section{Data Sharing Statement}

Data from non-clinical studies are outside of UCB's data sharing policy and are unavailable for sharing.

\section{Ethics Approval and Informed Consent}

Since this was a market research study, prior approval of the protocol by an ethics committee was not required. The 
study was conducted in accordance with relevant market research guidelines, including the obtaining of informed consent and adherence to ethical reporting standards.

\section{Acknowledgments}

The authors thank the patients, the investigators and their teams who took part in this study. The authors also acknowledge Wee Yan Ran, BSocSci (Hons), from Costello Medical, Singapore for medical writing and editorial assistance based on the authors' input and direction.

\section{Author Contributions}

Substantial contributions to study conception and design: JA, HG, JZ, AL, AJ; substantial contributions to analysis and interpretation of the data: JA, HG, JZ, AL, AJ; drafting the article or revising it critically for important intellectual content: JA, HG, JZ, AL, AJ; final approval of the version of the article to be published: JA, HG, JZ, AL, AJ; agreed on the journal to which the article will be submitted: JA, HG, $\mathrm{JZ}, \mathrm{AL}, \mathrm{AJ}$; agreed to take responsibility and be accountable for the contents of the article: JA, HG, JZ, AL, AJ.

\section{Funding}

This market research and article were sponsored by UCB Pharma. Support for third-party writing assistance for this article, provided by Wee Yan Ran from Costello Medical, Singapore, was funded by UCB Pharma in accordance with Good Publication Practice (GPP3) guidelines (http:// www.ismpp.org/gpp3). The authors acknowledge Costello Medical, Singapore for medical writing and editorial assistance based on the authors' input and direction.

\section{Disclosure}

JZ has received speaker honoraria from UCB, Lilly, Janssen-Cilag, Novartis and AbbVie. AL is an employee of UCB Pharma and participates in the UCB Stock Award Plan. JA has received sponsorship for conference attendance from UCB. AJ has received sponsorship for educational activities from UCB. The authors report no other conflicts of interest in this work.

\section{References}

1. Lyseng-Williamson KA. Certolizumab pegol administration devices: a profile of their use and usability. Drugs Ther Perspect. 2017;33 (11):515-522.

2. Hyrich KL, Verstappen SM. Biologic therapies and pregnancy: the story so far. Rheumatology. 2014;53(8):1377-1385.

3. Cimzia ${ }^{\mathbb{B}}$. (Certolizumab Pegol) $200 \mathrm{Mg} / \mathrm{Ml}$ Injection [Australian Prescribing Information]. Malvern: UCB Pharma; 2019.

4. Marengo MF, Suarez-Almazor ME. Improving treatment adherence in patients with rheumatoid arthritis: what are the options? Int J Clin Rheumtol. 2015;10(5):345-356.

5. van den Bemt BJ, Gettings L, Domańska B, Bruggraber R, Mountian I, Kristensen LE. A portfolio of biologic self-injection devices in rheumatology: how patient involvement in device design can improve treatment experience. Drug Delivery. 2019;26 (1):384-392.

6. Bailey K, Mountian I, Bruggraber R, Sunderland K, Tilt N, Szegvari B. Patient Satisfaction with CIMZIA $^{\circledR}$ (Certolizumab Pegol) AutoClicks ${ }^{\circledR}$ in the UK. Adv Ther. 2020;37(4):1522-1535.

7. Domańska B, Stumpp O, Poon S, Oray S, Mountian I, Pichon C. Using patient feedback to optimize the design of a certolizumab pegol electromechanical self-injection device: insights from human factors studies. Adv Ther. 2018;35(1):100-115.

8. Domańska B, VanLunen B, Peterson L, Mountian I, Schiff M. Comparative usability study for a certolizumab pegol autoinjection device in patients with rheumatoid arthritis. Expert Opin Drug Deliv. 2017; 14(1):15-22.

9. Keininger D, Coteur G. Assessment of self-injection experience in patients with rheumatoid arthritis: psychometric validation of the Self-Injection Assessment Questionnaire (SIAQ). Health Qual Life Outcomes. 2011;9(1):2.

10. Schiff M, Saunderson S, Mountian I, Hartley P. Chronic disease and self-injection: ethnographic investigations into the patient experience during treatment. Rheumatol Ther. 2017;4(2):445-463.

11. Maniadakis N, Toth E, Schiff M, et al. A targeted literature review examining biologic therapy compliance and persistence in chronic inflammatory diseases to identify the associated unmet needs, driving factors, and consequences. Adv Ther. 2018;35(9):1333-1355.

\section{Publish your work in this journal}

Patient Preference and Adherence is an international, peer-reviewed, open access journal that focusing on the growing importance of patient preference and adherence throughout the therapeutic continuum. Patient satisfaction, acceptability, quality of life, compliance, persistence and their role in developing new therapeutic modalities and compounds to optimize clinical outcomes for existing disease states are major areas of interest for the journal. This journal has been accepted for indexing on PubMed Central. The manuscript management system is completely online and includes a very quick and fair peer-review system, which is all easy to use. Visit http:// www.dovepress.com/testimonials.php to read real quotes from published authors. 